\title{
Effects of Videogame Distraction and a Virtual Reality Type Head-Mounted Display Helmet on Cold Pressor Pain in Young Elementary School-Aged Children
}

\author{
Lynnda M. Dahlquist, PHD, Karen E. Weiss, PHD, Emily F. Law, MA, Soumitri Sil, MA, Linda \\ Jones Herbert, MA, Susan Berrin Horn, MA, Karen Wohlheiter, MA, and Claire Sonntag Ackerman, PHD \\ Department of Psychology, University of Maryland, Baltimore County
}

\begin{abstract}
Objective This study examined the effects of videogame distraction and a virtual reality (VR) type headmounted display helmet for children undergoing cold pressor pain. Methods Fifty children between the ages of 6 and 10 years underwent a baseline cold pressor trial followed by two cold pressor trials in which interactive videogame distraction was delivered via a VR helmet or without a VR helmet in counterbalanced order. Results As expected, children demonstrated significant improvements in pain threshold and pain tolerance during both distraction conditions. However, the two distraction conditions did not differ in effectiveness. Conclusions Using the VR helmet did not result in improved pain tolerance over and above the effects of interactive videogame distraction without VR technology. Clinical implications and possible developmental differences in elementary school-aged children's ability to use VR technology are discussed.
\end{abstract}

Key words Acute pain; children; distraction; virtual reality technology.

Recent studies have shown that virtual reality (VR) technology can be used to provide pain-attenuating distraction for a variety of pediatric populations, including burn patients (Chan, Chung, Wong, Lien, \& Yang, 2007; Das, Grimmer, Sparnon, McRae, \& Thomas, 2005; Hoffman, Doctor, Patterson, Carrougher, \& Furness, 2000; van Twillert, Bremer, \& Faber, 2007), adolescents with cerebral palsy undergoing physical therapy (Steele et al., 2003), children undergoing IV placement for magnetic resonance imaging (MRI) or computed tomography (CT) scans (Gold, Kim, Kant, Joseph, \& Rizzo, 2006), and children with cancer undergoing portacatheter access (Gershon, Zimand, Lemos, Rothbaum, \& Hodges, 2003; Gershon, Zimand, Pickering, Rothbaum, \& Hodges, 2004; Wolitzky, Fivush, Zimand, Hodges, \& Rothbaum, 2005) and lumbar puncture (Wint, Eshelman, Steele, \& Guzetta, 2002). VR-assisted distraction also has been shown to improve children's tolerance of experimentally induced cold pressor pain (Dahlquist et al., 2007; Dahlquist et al., in press).

Although definitions of VR distraction in the literature vary considerably, most VR interventions involve a human-computer interface in which the user interacts with a responsive, multi-sensory virtual environment. Users typically wear a head-mounted display (HMD) helmet with integrated headphones that engages the visual and auditory sensory modalities and blocks external auditory and visual stimuli unrelated to the virtual environment. The user interacts with the virtual environment by manipulating a joystick or mouse, or via head- or hand-motion-sensitive tracking devices, resulting in a sense of being more or less "present" in the virtual environment (Regenbrecht, Schubert, \& Friedman, 1998; Schuemie, van der Straaten, Krijn, \& van der Mast, 2001).

The VR distraction interventions reported in the literature vary considerably in terms of the type of VR equipment used, ranging from simple 3D goggles (e.g., Chan et al., 2007; Wint et al., 2002) to expensive, technologically sophisticated helmets (Hoffman et al., 2006). The interventions also differ with regard to the nature of the VR environment and the content, quality, and responsiveness of the software, and the ways in which the participant interacts with the virtual environment, e.g., as a passive observer watching a movie

All correspondence concerning this article should be addressed to Lynnda M. Dahlquist, PHD, Department of Psychology, University of Maryland, Baltimore County, 1000 Hilltop Circle, Baltimore, MD 21250, USA.

E-mail: dahlquis@umbc.edu 
(e.g., Wint et al., 2002) versus actively engaging and altering the virtual environment through physical movements (e.g., Dahlquist et al., 2007). As a result of these methodological differences, it is difficult to evaluate the relative effectiveness of the various VR distraction strategies.

Study design issues also make it difficult to identify optimal intervention strategies. Most of the published VR distraction studies to date have compared VR distraction to a no-treatment control condition (e.g., Chan et al., 2007; Das et al., 2005; Gold et al., 2006; Steele et al., 2003; Wolitzky et al., 2005). Although these preliminary investigations provide encouraging support for the feasibility of using VR distraction for children experiencing acute clinical pain, such study designs do not allow one to determine if the observed beneficial effects on pain and distress were due to the use of VR technology or due to distraction effects that were unrelated to the VR technology. Of the studies comparing VR distraction to other types of distraction (such as videogames or movies), many also suffer from design flaws that limit the inferences that can be drawn regarding the specific effects of VR technology. For example, in several studies, the non-VR distraction interventions that were compared with the VR distraction interventions differed on several dimensions (e.g., the content and type of distraction activity) in addition to the use of VR technology, thus making the specific effects of the VR technology impossible to determine (e.g., Hoffman et al., 2000; Hoffman, Garcia-Palacios et al., 2001; van Twillert et al., 2007).

Few studies have tried to systematically isolate and examine the active ingredients of effective VR-assisted distraction interventions. Thus, little is known regarding the critical components of effective intervention. The limited literature available suggests that VR-assisted distraction during cold pressor exposure may be more effective when the distraction task is interactive (requiring the child to make some sort of response to the virtual environment), rather than passive (Dahlquist et al., 2007). However, the essential aspects of the VR equipment itself have not been determined.

To our knowledge, only three published studies have evaluated whether VR technology enhances videogame distraction for children. In a controlled case study (A-B-C-A) design, Gershon and colleagues (2003) observed an 8-yearold oncology patient undergoing portacatheter access during one baseline session with no distraction intervention, one port access in which he played a Virtual Gorilla game (Allison, Wills, Bowman, Wineman, \& Hodges, 1997) without VR equipment, one portacatheter access in which he played the Virtual Gorilla game with VR equipment (HMD helmet and handheld joystick), and a final baseline session. The VR condition resulted in substantially lower observed distress and lower nurse- and selfreported pain ratings compared to the baseline and non-VR videogame conditions. However, the applicability of these findings is limited by the use of a single subject and the reliance on a single observation for each intervention phase.

The only large-scale clinical study that specifically evaluated the effectiveness of VR-assisted videogame distraction compared to videogame play without VR technology was inconclusive (Gershon et al., 2004). Total Fifty-nine pediatric oncology patients aged 7-19 years undergoing port access were randomized to one of three groups: the Virtual Gorilla game played with the VR equipment, the Virtual Gorilla game played without the VR equipment, or standard care with no distraction. Children who used the VR equipment had lower pulse rates than children who received no distraction; however, they did not differ from children in the non-VR distraction condition on observed, self-reported, or physiological pain measures. Furthermore, no differences in observed pain behaviors were found between VR-distraction, non-VR distraction, and the no-distraction control conditions. However, the children in this study demonstrated very little distress at baseline, which likely limited their chances of demonstrating any improvement from the VR intervention.

Dahlquist et al. (2009) examined the utility of adding VR technology (an HMD helmet) to videogame distraction in a study of 41, 6- to 14-year-old children experiencing experimentally induced cold pressor pain in a laboratory setting. Using each subject as his/her own control, the visual and auditory nature of the distraction activity was held constant (all participants played a videogame in which the child scuba-dives in search of treasure chests in a $360^{\circ}$ virtual underwater environment) and the use of the HMD helmet was experimentally manipulated. As expected, both distraction conditions resulted in improved pain tolerance relative to baseline. However, only children older than 10 years of age demonstrated a significantly greater magnitude response with the VR helmet. The younger children appeared to benefit equally from distraction with or without the VR helmet.

Dahlquist et al. (2009) speculated that the differential response of the younger children to the VR helmet may be explained, in part, by the quality of the HMD helmet they used. Although the VFX3D Interactive Personal Display helmet (Interactive Imaging systems, Inc., Irvine, CA) could be adjusted to the child's inter-ocular measurements, the size of the helmet and the location of the earphones were not adjustable. Thus, the helmet may 
not have blocked out external stimuli as effectively for the younger, smaller children. Moreover, the helmet's visual display was slightly less vivid than the computer screen used in the non-VR distraction condition, which may have made it less appealing to younger children. Finally, the distraction software used by Dahlquist et al. (2009) may not have been optimally engaging for the younger children

The aim of the present study was to test the potential benefits of using a VR helmet to provide distraction during cold pressor exposure for the age group of children (i.e., 6- to 10-year-olds) that did not show significantly better performance with VR technology-enhanced distraction in Dahlquist et al. (2009). This modified replication of Dahlquist et al. (2009) also attempted to address methodological limitations of the previous study. Specifically, the present study utilized a VR HMD helmet that was lighter in weight, provided a larger field of view, and clearer picture in comparison to the equipment used in Dahlquist et al. (2009). Additionally, a videogame that was quick-paced and action-oriented was utilized in place of the relatively calm and relaxing videogame employed in the previous study. Finally, a slightly warmer water temperature was used $\left(7^{\circ} \mathrm{C}\right.$ compared to $\left.5^{\circ} \mathrm{C}\right)$ to minimize possible floor effects and allow for a wider range of pain tolerance responses from the children.

Using the same experimental design used by Dahlquist et al. (2009), each subject served as his/her own control. Following a baseline cold pressor trial, children underwent two distraction trials presented in random order, in which the visual and auditory nature of the distraction activity was held constant, and the use of the HMD helmet was experimentally manipulated. Children were expected to exhibit improvements in pain threshold and pain tolerance relative to baseline during both distraction conditions (with and without the VR helmet). The greatest improvements were expected when the children used the VR helmet.

\section{Methods \\ Participants}

Participants between the ages of 6 and 10 years were recruited from a university summer day camp. Parents enrolled their children in the study by approaching research staff at an information table near the camp check-in site. Of the 50 children who participated, 27 (54\%) were males, 20 (40\%) were Caucasians, 19 (38\%) were African Americans, 2 (4\%) were biracial, and 4 (8\%) were Asian/Pacific Islanders. Ethnicity was not reported for five participants. The mean age of participants was
8.14 years $(S D=1.38)$. Children were excluded from the study if exposure to cold temperatures was contraindicated (e.g., Raynaud's disease, sickle-cell disease), or if their parents reported they had mental retardation, hearing or vision impairments, vestibular difficulties, or motor disability that would interfere with using the VR equipment. However, no children were ineligible for the study based on these criteria.

\section{Materials and Equipment}

\section{Cold Pressor Apparatus}

Water temperature was maintained at $7^{\circ} \mathrm{C}\left( \pm 0.1^{\circ} \mathrm{C}\right)$ in a Thermo Electron Corporation Neslab RTE17 (Newington, $\mathrm{NH})$, refrigerated bath circulator $(60.0 \times 28.9 \times 47.9 \mathrm{~cm})$. In pilot testing, this water temperature elicited a range of pain tolerance scores with only minimal ceiling effects. Warmer water temperatures have been reported to cause greater problems with ceiling effects. For example, $93 \%$ of 10- to 14-year-old subjects demonstrated ceiling effects (tolerated the full $4 \mathrm{~min}$ ) at a water temperature of $10^{\circ} \mathrm{C}$ (Goodman \& McGrath, 2003). Similar ceiling effects using warmer water have been reported by other investigators (e.g., Miller, Barr, \& Young, 1994; Piira, Taplin, Goodenough, \& von Baeyer, 2002; von Baeyer, Piira, Chambers, Trapanotto, \& Zeltzer, 2005).

\section{Thermal Feedback System}

Finger temperature was measured at baseline and between each trial using a Thermal Feedback System, manufactured by Bio-feedback Systems, Inc., Boulder, CO (Model DT-100; Power ID-91).

\section{Videogame Software}

The Ice Age 2: The Meltdown ${ }^{\odot}$ "Eviscerator" game (Twentieth Century Fox, Sierra Entertainment) was utilized as the interactive distraction activity. The "Eviscerator" game segment provides a third-person perspective of a $360^{\circ} 3 \mathrm{D}$ virtual environment in which the participant controls "Sid the Sloth" who slides down a snowy path while trying to collect acorns and avoid obstacles. The soundtrack provides upbeat, quick-tempo background music and sounds of "Sid the Sloth" riding down the path.

\section{Computer}

The videogame was generated by a Dell Dimension 8400 desktop computer with a 19-inch monitor screen (1024 × 768 resolution; Round Rock, TX), a Radeon X850XT, Platinum Edition video card, and integrated 7.1 channel audio speakers. The computer was placed approximately 1 meter from the child. The use of a Logitech (Freemont, CA) Freedom 2.4 cordless joystick allowed 
children to move the joystick with their dominant hand while the other hand was in the cold water.

\section{Virtual Reality Equipment}

An adjustable HMD helmet with integrated headphones (5DT HMD Interactive Personal Display System; Fifth Dimension Technologies, Irvine, CA) was connected to the Dell Dimension 8400 desktop computer. The stereoscopic 1.44 million pixel videogame color display with a 26 degree viewing angle was projected through the goggles in the HMD upon connection; the goggles were adjusted to the individual's inter-ocular distance in order to reduce eye strain. Auditory effects of the game were delivered via the integrated Senheiser HD25 ( $16 \mathrm{~Hz}$ to $22 \mathrm{kHz}$ ) headphones in the helmet. Children wore a disposable surgical cap under the helmet for sanitary reasons.

\section{Measures}

\section{Pain-dependent Variables}

Pain threshold was defined as the elapsed time (in seconds) from hand submersion in the cold water until the child's first report of pain. Pain tolerance was defined as the total time (in seconds) that the child's hand remained submerged in the cold water.

\section{Post-study Qualitative Questionnaire}

Participants were asked the following open-ended questions about the distraction plus helmet condition after completing both distraction trials: "When you were wearing the helmet, what kind of noise (other than what was in the game) did you hear while you were playing Ice Age 2?", "When you were wearing the helmet, what other things did you see (other than the game) while you were playing Ice Age 2?", in order to determine whether the participants noticed external visual or auditory stimuli while wearing the helmet and playing the game.

\section{Procedure}

\section{Consent}

The study was approved by the University Institutional Review Board. Informed consent was obtained from the parent at the time of recruitment (camp participants) or when the parent brought the child to the lab. Assent was obtained from the child prior to conducting experimental procedures. In addition to explaining study procedures, the consenting experimenter also discussed at length the child's right to refuse to participate or to discontinue the study at any time without any negative consequences and probed the child's understanding of these concepts before proceeding with the study.

\section{Design}

Participants underwent a baseline cold pressor trial followed by two cold pressor trials in which distraction with the VR helmet (distraction plus helmet) or distraction without the VR helmet (distraction-only) was presented in counterbalanced order. Visual and auditory distracting stimuli in both the distraction plus helmet condition and the distraction-only condition were the same; only the use of the helmet varied in the two conditions. During the distraction plus helmet condition, participants used a joystick to play a videogame that was presented through a 3D HMD helmet with integrated headphones. During the distraction-only condition, participants played the same videogame while it was presented via a computer screen and stereo speakers. The amount of time that elapsed until the child reported pain (pain threshold) and total time the child kept his/her hand in the cold water (pain tolerance) were measured in each cold pressor trial.

A subgroup of participants $(n=14)$ underwent two baseline cold pressor trials before participating in the two distraction conditions in order to allow for the examination of the effects of repeated exposure to the cold pressor. Using the urn randomization method described by Wei and Lachin (1988), children were stratified by age and gender and randomly assigned to one of the following groups: (a) single trial baseline, distraction-only first, (b) single trial baseline, distraction plus helmet first, (c) two-trial baseline, distraction-only first; and (d) twotrial baseline, distraction plus helmet first.

\section{Setting}

The study was conducted in a $4.88 \mathrm{~m} \times 3.66 \mathrm{~m}$ carpeted laboratory room that was maintained at a temperature between 21 and $22^{\circ} \mathrm{C}$. Two graduate or undergraduate psychology student experimenters were present during each trial. Parents were not present.

\section{Cold Pressor Trials}

Before the first trial, the experimenter taped the temperature sensor to the index finger of the child's non-dominant hand, and recorded the child's finger temperature after a 1 min adaptation period. After the probe was removed, the experimenter read the child the following instructions:

In a minute, you will be asked to place one of your hands in this water. This water is very cold. You will notice that after a while your hand will start to feel uncomfortable or hurt. At this time please say "It hurts now" so that I know when your hand starts to feel this way. Eventually you will decide that you can no longer keep your hand in the water because it is 
too uncomfortable or hurts too much. That's okay, because when you feel this way you should remove your hand from the water.

To probe the child's understanding of the directions, the experimenter asked the following questions prior to each cold pressor trial: "What are you going to say when your hand starts to hurt?" and "What should you do when you don't want to keep your hand in the water any longer?" Any confusion about the directions or the child's freedom to terminate the trial at any time was addressed by the experimenter prior to beginning the study. These probes were repeated prior to each cold pressor trial. None of the participants asked to terminate the study.

The baseline cold pressor trial was then conducted. The child was seated so that the non-dominant arm could be comfortably extended downward at his/her side with the hand placed in the circulating water bath to wrist level. Timing began as soon as the participant's hand was submerged to wrist level and ended when the hand was removed. The time at which the child said, "It hurts now" (pain threshold) and the total amount of time the hand was submerged (pain tolerance) were recorded. At the end of the trial, the child's hand was placed in a warm water bath $\left(32^{\circ} \mathrm{C}\right)$ for approximately $5 \mathrm{~min}$, and warmed to within $1{ }^{\circ} \mathrm{C}$ of the baseline temperature.

Before the first distraction trial, the experimenter explained the Ice Age 2: The Meltdown ${ }^{(\mathcal{C}}$ "Eviscerator" videogame. The participant was instructed to collect acorns while passing through the red arches. The experimenter then modeled the correct use of the joystick, and ensured that the child understood how to operate the joystick, navigate the character, and collect acorns by allowing him/her to play through the entire Ice Age 2: The Meltdown ${ }^{\odot}$ "Eviscerator" game segment (for approximately $3.5 \mathrm{~min}$ ) before the first experimental trial.

\section{Distraction Plus Helmet Condition}

The participant was told that he/she would be playing the Ice Age 2: The Meltdown ${ }^{\odot}$ "Eviscerator" videogame while wearing the VR helmet and using the cordless joystick, while his/her non-dominant hand was placed in the cold water. The experimenter explained that he/she would see the videogame through the viewer in the helmet and hear the game through the earphones in the helmet, and that the game would end when he/she removed the hand from the water. The VR helmet was then placed on the child's head and the game was started. After $10 \mathrm{~s}$ of play to allow the child to become engaged in the game, the child's non-dominant hand was placed in the cold pressor. All other procedures were identical to the baseline trial.

\section{Distraction-only Condition}

Procedures in the distraction-only condition were identical to the distraction plus helmet condition with the following exception: the child was told that he/she would be playing the Ice Age 2: The Meltdown ${ }^{\mathcal{O}}$ "Eviscerator" game while viewing the game on the computer screen and hearing the game through the computer speakers.

After the final distraction trial, participants completed a brief qualitative questionnaire about the VR experience and the videogame. They also were asked whether they heard or saw anything other than the videogame while wearing the VR helmet and to describe the extraneous sounds or sights they noticed. The number of children who reported seeing any extraneous stimuli and the number who reported hearing any extraneous stimuli during the distraction plus helmet trial were recorded. Children then selected a prize from a bag of trinkets (e.g., key chains, pens, and bracelets valued under $\$ 2.00$ ) and received a $\$ 10.00$ gift card.

\section{Results \\ Preliminary Analyses}

\section{Descriptive Analyses}

First, descriptive analyses were conducted on all variables of interest to determine if the data were normally distributed. The distributions of the pain tolerance and pain threshold scores for the three conditions (baseline, distraction-only, and distraction plus helmet) met criteria for substantial positive skew and kurtosis (Tabachnick \& Fidell, 2001). LOG-10 transformations produced skew and kurtosis values nearest to zero; therefore, all subsequent analyses were conducted with LOG-10 transformed pain tolerance and pain threshold scores.

Baseline pain tolerance scores ranged from 5.67 to $62.00 \mathrm{~s}$, with a mean of $20.75 \mathrm{~s},(S D=11.09 \mathrm{~s})$. Baseline pain threshold scores ranged from 3.40 to $41.88 \mathrm{~s}$ with a mean of $14.38 \mathrm{~s}(S D=8.36 \mathrm{~s})$. After transformation, baseline pain tolerance scores ranged from 0.75 to 1.79 , with an overall mean of $1.26(S D=.23)$. Transformed baseline pain threshold scores ranged from 0.53 to 1.62 , with an overall mean of $1.09(S D=.25)$.

\section{Habituation}

Participants who underwent two baseline trials showed no evidence of habituation to the cold pressor tasks. Their pain tolerance scores showed no significant change from Trial 1 to Trial $2(M=1.16, S D=.18$ vs. $M=1.18$, $S D=.18, p=.39$ ). Similarly, their pain threshold scores did not change significantly from Trial 1 to Trial 2 $(M=.98, S D=.25$ vs. $M=1.02, S D=.23, p=.18)$. 


\section{Order Effects}

Four mixed model $2 \times 2$ (order by trial) analyses of variance (ANOVAs) were conducted to determine whether the order in which children participated in the experimental conditions affected their pain threshold and pain tolerance scores. For each analysis, the between-subjects variable was order of presentation of the experimental condition (i.e., first or second experimental trial) and the within-subjects variable was trial (i.e., last baseline vs. experimental). Pain threshold and pain tolerance scores were examined separately for each experimental condition. Results indicated that neither pain threshold nor pain tolerance scores significantly differed as a result of the order in which the distraction plus helmet condition was presented or the order in which the distraction-only condition was presented (all $F s \leq 2.36$, all $p s>.13$ ). Therefore, for subsequent analyses, the data were collapsed across order.

\section{Relative Effectiveness of Distraction With and Without the HMD Helmet}

Two separate within-subjects ANOVAs were conducted to compare each participant's pain tolerance and pain threshold scores across the three conditions (the child's last baseline trial, distraction-only trial, and distraction plus helmet trial). Results indicated significant effects for experimental condition for pain tolerance $[F(2,98)=$ 20.45, $\left.p<.001, f^{2}=.65\right]$ and for pain threshold $\left[F(2,70)=21.84, p<.001, f^{2}=.79\right]$.

Post-hoc paired t-tests with Bonferroni correction indicated that children demonstrated significantly higher pain tolerance scores during the distraction-only condition $[M=1.45, S D=.33, t(49)=6.01, p<.001]$, and the distraction plus helmet condition $[M=1.44, S D=.38$, $t(49)=4.68, p<.001]$, when compared to their baseline scores $(M=1.25, S D=.23)$. However, the pain tolerance scores obtained in the two distraction conditions did not differ significantly, $t(49)=.11, p>.91$.

Similar results were found for pain threshold scores. Children's pain threshold scores were significantly higher during the distraction-only condition $[M=1.28, S D=.23$, $t(39)=6.89, p<.001]$ and the distraction plus helmet condition $[M=1.22, S D=.30, t(37)=3.32, p<.002]$ than during baseline $(M=1.09, S D=.26)$. The pain threshold scores of the two distraction conditions did not differ significantly, $t(40)=1.93, p>.05$ (Figure 1 ).

\section{Analysis of Post-Study Qualitative Questionnaire}

A total of 44 participants (88\%) reported that they did not see anything other than the game and the inside of the helmet when wearing the helmet. The remaining six participants (12\%) reported seeing one or two of the following visual stimuli: the computer stand, the wall, a filing cabinet, or people. Thirty-six participants $(72 \%)$ reported that they did not hear anything other than the game when wearing the helmet. The remaining 14 (28\%) participants reported hearing one of the following auditory stimuli: the cold pressor, people talking, the joystick moving, footsteps, and noise from the hall. Participants' pain threshold and pain tolerance scores during the distraction plus helmet condition did not significantly differ in relation to whether or not they heard or saw any external stimuli while playing the videogame (all $p s>.05$ ).

\section{Discussion}

The results of this study replicate the findings of Dahlquist et al. (2009). Compared to their baseline performance, the 6- to 10-year-old children in this study demonstrated

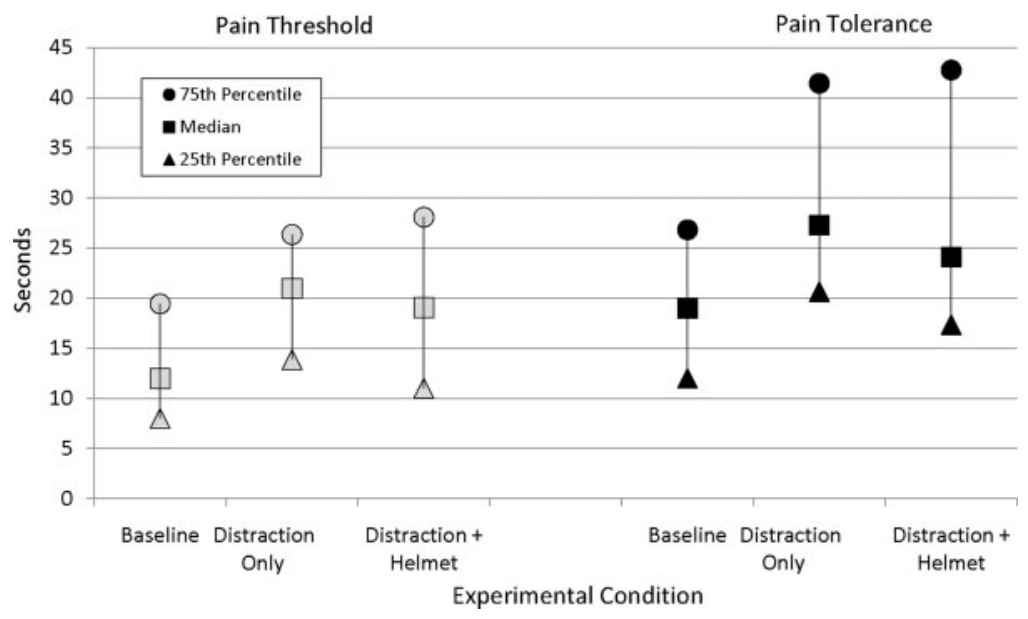

Figure 1. Medians and inter-quartile ranges for untransformed pain threshold and pain tolerance scores across experimental conditions $(n=50)$. 
significant improvements in cold pressor pain tolerance during interactive videogame distraction. Similar to the findings in past studies (Dahlquist et al., 2007; Dahlquist et al., 2009), the magnitude of this effect was large, according to Cohen's (1969) criteria; on average, children tolerated the cold water exposure 18-22 seconds longer during the interactive distraction conditions. These findings suggest that videogame distraction may prove to be a very effective pain management intervention for young elementary school-aged children. If similar findings were obtained in a clinical setting, even this relatively brief improvement in pain tolerance could be clinically significant. Twenty additional seconds of pain tolerance could allow additional time to access a difficult vein or a tender port site, to cleanse a wound, administer an intramuscular injection, or change a dressing.

The study findings also replicate the findings of Dahlquist et al. (2009) with respect to the absence of a differential response to interactive distraction with or without the enhancement of VR technology in children between the ages of 6 and 10 years undergoing laboratory pain. The use of this particular VR helmet did not enhance their pain tolerance over and above the benefits accrued by engaging in the interactive videogame distraction task. This finding suggests a number of possible explanations that warrant further study.

First, although the VR helmet used in this study was superior to the VR helmet used by Dahlquist et al. (2009) in terms of resolution and vividness, it still may have qualities that make it unsuitable or less than optimal for children in the 6- to 10-year-old age range. First, the field of view provided by the HMD measured only 26 degrees diagonal. Recent work by Hoffman et al. (2006) with adults experiencing experimentally induced thermal pain suggests that a greater magnitude improvement in subjective ratings of pain sensations can be achieved with a helmet that includes a wider field of view (i.e., 65 degree vs. 35 degree diagonal). The importance of field of view for children has not been studied. Although the helmet used in the present study was of mid-range quality (approximately $\$ 4000$ retail cost) and comparable to equipment used in past studies, it may have been possible to obtain better results with a helmet with a wider field of view. However, helmets of this quality typically cost about $\$ 30,000$ and consideration needs to be given to the practicality of using such equipment in a clinical setting.

The basis for these apparent developmental differences in children's ability to use certain types of VR technology has not yet been studied. It has long been assumed that VR interventions require a suspension of awareness of the
VR equipment and the external environment in favor of attention to the virtual environment (Schubert, Friedmann, \& Regenbrecht, 2001; Schuemie, van der Straaten, Krijn, \& van der Mast, 2001; Witmer \& Singer, 1998). For the VR helmet used in the present study, the child had to focus attention on the approximately $6.35 \times 6.35 \mathrm{~cm}$ screen in the center field of view and ignore the black areas that surrounded the screen, as well as ignore any visual stimuli evident through the open area of the helmet below the viewer (e.g., one's arms, clothing, the joystick, the floor). Although the specific attentional processes that facilitate the use of VR distraction have not yet been studied, it would seem reasonable to assume that selective attention, i.e., the ability to focus on necessary information in the environment and exclude less relevant information (Brodeur, Trick, $\&$ Enns, 1997), plays an important role. Children under 10 years of age, who do not yet have fully developed attention regulation abilities (Betts, Mckay, Maruff, \& Anderson, 2006; Smith, Kemler, \& Arnonfreed, 1975) would be expected to find the process of ignoring stimuli other than the HMD screen more difficult than would older children. Future studies should examine the specific attentional skills that may moderate children's responses to VR distraction.

Strengths of this study include the diversity of the sample (60\% were non-Caucasian), which contributes to the literature regarding pain management in diverse populations and improves the generalizability of the findings. In addition, the tightly controlled experimental design allowed for a clear test of the potential benefit of using a VR helmet for acute cold pressor pain.

\section{Limitations and Future Directions}

The primary limitation of this study involves the experimental nature of the pain stimulus that was studied. The children in this study were in complete control of the amount of time they were exposed to the painful stimulus, whereas children experiencing painful medical care often have much less or little control over the duration of the pain stimulus. Thus, one cannot determine whether the same pattern of results would be obtained with acute clinical pain. It is possible that videogame distraction would be less effective in a clinical setting or less effective for children with a history of aversive pain experiences.

On the other hand, it is possible that the VR helmet may help children ignore extraneous stimuli in the clinical environment that might otherwise interfere with focusing their attention on a videogame or other type of therapeutic distractor. The laboratory setting of this study was 
designed to be free of unintended distractions; children did not have to filter out many extraneous stimuli (other than the pain stimulus) in order to focus attention on the videogame distraction. As evidenced by the post-trial qualitative probes, few children reported noticing anything other than the videogame stimuli while they were wearing the VR helmet. However, the clinical medical setting is much more stimulating, with many competing sights and sounds, which may make it harder for children to focus attention on any distraction stimulus. Further research is needed to test whether a VR helmet mutes or blocks children's perceptions of extraneous environmental stimuli and thereby facilitates children's selective attention to the distraction stimuli, and whether improved pain management results. To do so, future studies should assess the child's awareness of external stimuli and/or engagement with the distractor during distraction trials conducted with a VR helmet as well as during distraction conducted without the use of a VR helmet. Such data also will help determine the degree to which using VR technology is cost effective.

Finally, the results obtained in this study may not generalize to findings with other types of VR equipment. There are many possible ways to employ VR technology and many different types of VR equipment and software. Given that the utility of VR distraction may well depend on the technical specifications of the VR equipment, future studies of VR distraction should, at the very least, precisely specify the technical characteristics of the devices used to create the VR environment. Rather than focus on whether or not VR distraction is effective, future studies should examine what types of VR equipment and what types of distraction activities are most effective for children of various ages, experience, and preferred coping strategies and for what types of pain stimuli.

\section{Acknowledgments}

The authors thank Jerry Prieto and the UMBC Summer Day Camp staff for arranging the day camp schedule so that the campers could participate in this study, and Monica Jimeno, Joseph Keller, Michael Miller, Cyrus Mistry, Megan Sipes, Rebecca Stern, and Jessica Wentling for helping with participant recruitment, experimental procedures, and data management.

\section{Funding}

Grant No. R01HD050385 from the National Institutes of Health, National Institute for Child Health and Development.
Conflict of interest: None declared.

Received April 7, 2009; revisions received August 7, 2009; accepted August 17, 2009

\section{References}

Allison, D., Wills, B., Bowman, D., Wineman, J., \& Hodges, L. F. (1997). The virtual reality gorilla exhibit. IEEE Computer Graphics and Applications, 17, 30-38.

Betts, J., Mckay, J., Maruff, P., \& Anderson, V. (2006). The development of sustained attention in children: The effect of age and task load. Child Neuropsychology, 12, 205-221.

Brodeur, D., Trick, L., \& Enns, J. (1997). Selective attention over the lifespan. In J. A. Burack, \& J. T. Enns (Eds.), Attention, development, and psychopathology (pp. 74-94). New York: Guilford.

Chan, E. A., Chung, J. W. Y., Wong, T. K. S., Lien, A. S. Y., \& Yang, J. Y. (2007). Application of a virtual reality prototype for pain relief of pediatric burn in Taiwan. Journal of Clinical Nursing, 16, 786-793.

Cohen, J. (1969). Statistical power analysis for the behavioral sciences. New York: Academic Press.

Dahlquist, L. M., McKenna, K. D., Jones, K. K., Dillinger, L., Weiss, K. E., \& Ackerman, C. S. (2007). Active and passive distraction using a head-mounted display helmet: Effects on cold pressor pain in children. Health Psychology, 26, 794-801.

Dahlquist, L. M., Weiss, K. E., Clendaniel, L. D., Law, E. F., Ackerman, C. S., \& McKenna, K. M. (2009). Effects of videogame distraction using a virtual reality type head-mounted display helmet on cold pressor pain in children. Journal of Pediatric Psychology, 34, 574-584

Das, D. A., Grimmer, K. A., Sparnon, A. L., McRae, S. E., $\&$ Thomas, B. H. (2005). The efficacy of playing a virtual reality game in modulating pain for children with acute burn injuries: A randomized controlled trial. BMC Pediatrics, 5, 1-10.

Gershon, J., Zimand, E., Lemos, R., Rothbaum, B. O., $\&$ Hodges, L. (2003). Use of virtual reality as a distractor for painful procedures in a patient with pediatric cancer: A case study. CyberPsychology and Behavior, 6, 657-661.

Gershon, J., Zimand, E., Pickering, M., Rothbaum, B. O., $\&$ Hodges, L. (2004). A pilot and feasibility study of virtual reality as a distraction for children with cancer. 
Journal of the American Academy of Child and Adolescent Psychology, 43, 1243-1249.

Gold., J. I., Kim, S. H., Kant, A. J., Joseph, M. H., $\&$ Rizzo, A. (2006). Effectiveness of virtual reality for pediatric pain distraction during IV placement. CyberPsychology and Behavior, 9, 207-212.

Goodman, J. E., \& McGrath, P. J. (2003). Mothers' modeling influences on children's pain during cold pressor task. Pain, 104, 559-565.

Hoffman, H. G., Doctor, J. N., Patterson, D. R., Carrougher, G. J., \& Furness, T. A. III (2000). Virtual reality as an adjunctive pain control during burn wound care in adolescent patients. Pain, 85, 305-309.

Hoffman, H., Garcia-Palacios, A., Patterson, D. R., Jensen, M., Furness, T. A. III, \& Ammons, W. F. Jr. (2001). The effectiveness of virtual reality for dental pain control: A case study. CyberPsychology and Behavior, 4, 527-535.

Hoffman, H. G., Seibel, E. J., Richards, T. L., Furness, T. A. III, Patterson, D. R., \& Sharar, S. R. (2006). Virtual reality helmet display quality influences the magnitude of virtual reality analgesia. The Journal of Pain, 7 , 843-850.

Miller, A., Barr, R. G., \& Young, S. N. (1994). The cold pressor test in children: Methodological aspects and the analgesic effect of intraoral sucrose. Pain, 56, 175-183.

Piira, T., Taplin, J. E., Goodenough, B., \& von Baeyer, C. (2002). Cognitive-behavioral predictors of children's tolerance of laboratory-induced pain: Implications for clinical assessment and future directions. Behaviour Research and Therapy, 40, 571-584.

Regenbrecht, H., Schubert, T., \& Friedman, F. (1998). Measuring the sense of presence and its relations to fear of heights in virtual environments. International Journal of Human-Computer Interaction, 10, 233-249.

Schuemie, M., van der Straaten, P., Krijn, M., \& van der Mast, C. (2001). Research on presence in virtual reality: A survey. CyberPsychology and Behavior, 4, 183-201.

Smith, L. B., Kemler, D. G., \& Arnonfreed, J. (1975). Developmental trends in voluntary selective attention: Differential effects of source distinctiveness. Journal of Experimental Child Psychology, 20, 352-365.

Steele, E., Grimmer, K., Thomas, B., Mulley, B., Fulton, I., \& Hoffman, H. (2003). Virtual reality as a pediatric pain modulation technique: A case study. CyberPsychology and Behavior, 6, 633-638.

Tabachnick, B. G., \& Fidell, L. S. (2001). Using multivariate statistics (4th ed.). Mahwah, N.J.: Erlbaum.

van Twillert, B., Bremer, M., \& Faber, A. W. (2007). Computer-generated virtual reality to control pain and anxiety in pediatric and adult burn patients during wound dressing changes. Journal of Burn Care and Research, 28, 694-702.

von Baeyer, C. L., Piira, T., Chambers, C. T., Trapanotto, M., \& Zeltzer, L. K. (2005). Guidelines for cold pressor as an experimental pain stimulus for use with children. The Journal of Pain, 6, 218-227.

Wei, L. J., \& Lachin, J. M. (1988). Properties of the urn randomization in clinical trials. Controlled Clinical Trials, 9, 345-364.

Wint, S. S., Eshelman, D., Steele, J., \& Guzzetta, C. E. (2002). Effects of distraction using virtual reality glasses during lumbar punctures in adolescents with cancer. Oncology Nursing Forum, 29, E8-E15.

Witmer, B. G., \& Singer, M. J. (1998). Measuring presence in virtual environments: A presence questionnaire. Presence, 7(3), 225-240.

Wolitzky, K., Fivush, R., Zimand, E., Hodges, L., $\&$ Rothbaum, B. O. (2005). Effectiveness of virtual reality distraction during a painful medical procedure in pediatric oncology patients. Psychology and Health, 20, 817-824. 